\title{
Induction of protein aggregation and starvation response by tRNA modification defects
}

\author{
Roland Klassen $^{1}$ (D) Alexander Bruch ${ }^{1} \cdot$ Raffael Schaffrath $^{1}$ (i)
}

Received: 29 July 2020 / Revised: 17 August 2020 / Accepted: 18 August 2020 / Published online: 29 August 2020

(C) The Author(s) 2020

\begin{abstract}
Posttranscriptional modifications of anticodon loops contribute to the decoding efficiency of tRNAs by supporting codon recognition and loop stability. Consistently, strong synthetic growth defects are observed in yeast strains simultaneously lacking distinct anticodon loop modifications. These phenotypes are accompanied by translational inefficiency of certain mRNAs and disturbed protein homeostasis resulting in accumulation of protein aggregates. Different combinations of anticodon loop modification defects were shown to affect distinct tRNAs but provoke common transcriptional changes that are reminiscent of the cellular response to nutrient starvation. Multiple mechanisms may be involved in mediating inadequate starvation response upon loss of critical tRNA modifications. Recent evidence suggests protein aggregate induction to represent one such trigger.
\end{abstract}

Keywords tRNA modification $\cdot$ Protein aggregation $\cdot$ Decoding $\cdot$ Starvation response

\section{Background}

During decoding of mRNA, codons are recognized by the tRNA anticodon. For efficient decoding, the tRNA must be correctly folded into an L-shaped structure and the anticodon presented in an unpaired open loop. Posttranscriptional modifications in the anticodon loop are thought to improve codon recognition and contribute to anticodon loop stability by promoting base stacking interactions, reducing the flexibility of the sugar phosphate backbone and preventing unwanted across-the-loop base pairing (Agris 2008; Sokołowski et al. 2017; Väre et al. 2017; Vendeix et al. 2012). For example, tRNA ${ }_{\text {UUU }}^{\text {Lys }}$ contains $\mathrm{mcm}^{5} \mathrm{~s}^{2} \mathrm{U}_{34}$ (5-methoxycarbonylmethyl-2-thiouridine at position 34) and ct ${ }^{6} \mathrm{~A}_{37}$ (cyclic $N^{6}$-threonylcarbamoyladenosine at position 37) modifications which each fulfill one or more of these tasks (Johansson et al. 2018; Miyauchi et al. 2013; Schaffrath and Leidel 2017; Thiaville et al. 2014). Both, $\mathrm{mcm}^{5} \mathrm{~s}^{2} \mathrm{U}$ and $\mathrm{ct}^{6} \mathrm{~A}$ are formed by multiple biosynthetic enzymes and steps. Completion of $\mathrm{mcm}^{5} \mathrm{~s}^{2} \mathrm{U}$

Communicated by M. Kupiec.

Roland Klassen

roland.klassen@uni-kassel.de

1 Institut für Biologie, Fachgebiet Mikrobiologie, Universität Kassel, Heinrich-Plett-Str. 40, 34132 Kassel, Germany synthesis is abolished at distinct steps in elp 3 and urm 1 mutants, while $\mathrm{ct}^{6} \mathrm{~A}$ formation from the $\mathrm{t}^{6} \mathrm{~A}$ ( $N^{6}$-threonylcarbamoyladenosine) precursor requires TCDl (Huang et al. 2005; Leidel et al. 2009; Miyauchi et al. 2013). Hence, in elp3, urm 1 and $t c d 1$ mutants, distinct pathway intermediates are formed at the target nucleosides $\mathrm{U}_{34}$ and $\mathrm{A}_{37}$. Consistent with functional redundancy, joint abrogation of $\mathrm{mcm}^{5} \mathrm{~s}^{2} \mathrm{U}$ synthesis at different steps and prevention of $t^{6} \mathrm{~A}$ to $\mathrm{ct}^{6} \mathrm{~A}$ conversion results in a functional defect of tRNA ${ }_{\text {UUU }}^{\text {Lys }}$ normally carrying these modifications (Klassen et al. 2016). A similar functional redundancy exists in the tRNA ${ }_{\text {UUG }}^{\mathrm{Gln}}$ anticodon loop which naturally carries $\mathrm{mcm}^{5} \mathrm{~s}^{2} \mathrm{U}$ and $\Psi_{38}$ (pseudouridine at position 38) (Han et al. 2015; Klassen et al. 2016). Combined absence of $\mathrm{mcm}^{5} \mathrm{~s}^{2} \mathrm{U}$ and $\Psi_{38}$ in elp $3 \mathrm{deg} 1$ or urm 1 degl double mutants causes a severe functional impairment of this tRNA. When formation of $\mathrm{mcm}^{5} \mathrm{~s}^{2} \mathrm{U}$ is completely abolished by combining elp 3 and urm 1 or elp 6 and $n c s 2$ modifications, both, tRNA ${ }_{\text {UUG }}^{\text {Gln }}$ and tRNA ${ }_{\text {UUU }}^{\text {Lys }}$ are functionally impaired (Björk et al. 2007; Klassen et al. 2015; Nedialkova and Leidel 2015; Xu et al. 2019). 


\section{Effects of modification loss on decoding and protein homeostasis}

In the mutants carrying combinations of tRNA modification defects, negative phenotypes and translational incompetence are routinely suppressed by overexpression of the functionally impaired tRNAs (Björk et al. 2007; Han et al. 2015; Klassen et al. 2015, 2016; Nedialkova and Leidel 2015). Elevated abundance of the hypomodified tRNA is thought to counteract the translational deficiency, which may result from increased rejection rate during the codon recognition process (Ranjan and Rodnina 2017; Rezgui et al. 2013). Another cellular consequence of such specific tRNA defects is a severe protein homeostasis disturbance, resulting in the accumulation of protein aggregates (Fig. 1) (Nedialkova and Leidel 2015). The exact mechanism how combined tRNA modification defects trigger protein aggregation is not known, but it can be assumed that ribosomal pausing is an important factor for this effect. Ribosomal pausing at CAA (Gln) and AAA (Lys) codons has been indeed demonstrated for yeast strains lacking $\mathrm{mcm}^{5} \mathrm{~s}^{2} \mathrm{U}$ (Nedialkova and Leidel 2015), and $\mathrm{mcm}^{5} \mathrm{~s}^{2} \mathrm{U}$ deficiency in combination with either loss of $\mathrm{ct}^{6} \mathrm{~A}$ or $\Psi_{38}$ likely aggravates pausing at CAA or AAA codons, respectively (Bruch et al. 2020; Klassen et al. 2016;

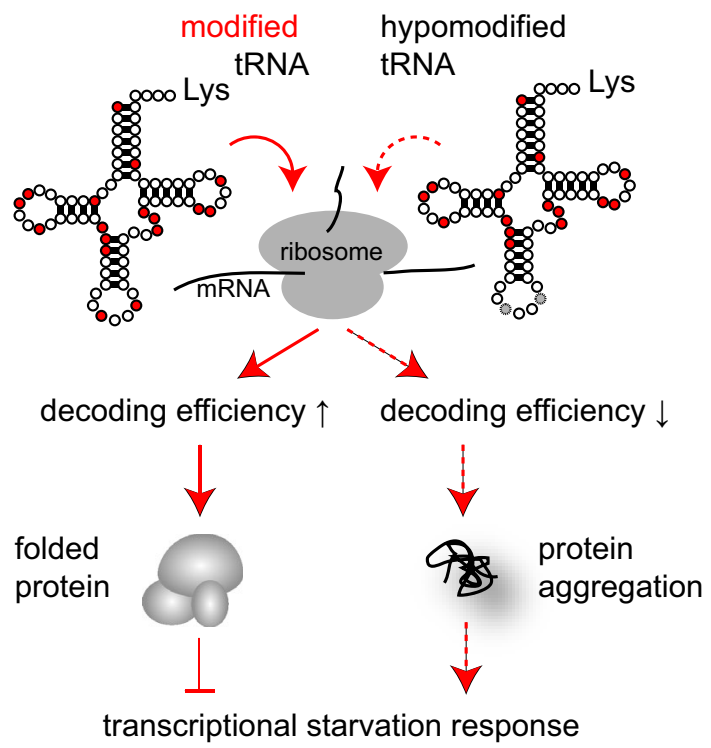

Fig. 1 Model for the induction of a transcriptional starvation response in combined absence of anticodon loop modifications. RRNA $_{\text {UUU }}^{\text {Lys }}$ depicted with modified positions (indicated in red). In combined elp3 tcdl or urml tcdl mutants, anticodon loop modifications $\mathrm{mcm}^{5} \mathrm{~s}^{2} \mathrm{U}$ and $c t^{6} \mathrm{~A}$ are missing (indicated in grey), causing decreased decoding efficiency of cognate AAA (Lys) codons. Multiple mechanisms are discussed how such decoding defect may cause accumulation of cellular protein aggregates. New results suggest that protein aggregates are involved in triggering a subsequent transcriptional response reminiscent of nutrient starvation
Pollo-Oliveira et al. 2020). Such pause during translational elongation may as well increase the occurrence of ribosomal errors including +1 frameshifts and, potentially, mistranslation due to misreading by near- or non-cognate tRNAs. An increase in +1 frameshift rates has in fact been detected in yeast strains lacking $\mathrm{mcm}^{5} \mathrm{~s}^{2} \mathrm{U}$ alone and in combination with ct $^{6} \mathrm{~A}$ defects (Klassen et al. 2017; Pollo-Oliveira et al. 2020; Tükenmez et al. 2015). While in such mutants, the efficiency of near/non-cognate tRNA misincorporation at the AAA or CAA codons has not been investigated, a similar effect was observed for misreading of CGC (Arg) codons by tRNA $^{\text {His }}{ }_{\text {GUG }}$ (Khonsari and Klassen 2020). Here, absence of Pus1 dependent $\Psi$ in the normal CGC decoder tRNA ${ }_{\text {ICG }}^{\text {Arg }}$ increased misreading by $\mathrm{RNA}_{\mathrm{GUG}}^{\mathrm{His}}$, which is not naturally modified by Pus1. Thus, in general, impairment of a cognate tRNA upon loss of critical modifications may increase near cognate misreading by a competitor tRNA that does not rely on the same modification. In addition to effects potentially associated with ribosomal pausing, the ability of the hypomodified tRNA itself to engage in misreading might also be affected by loss of critical anticodon loop modifications. Some specific errors indeed increased in the absence of $\mathrm{mcm}^{5} \mathrm{~s}^{2} \mathrm{U}$ (Joshi et al. 2018). However, when misreading of near cognate codons with wobble base mismatches to tRNA ${ }_{\text {UUU }}^{\text {Lys }}$ was studied, $\mathrm{mcm}^{5} \mathrm{~s}^{2} \mathrm{U}$ promoted rather than inhibited these types of errors (Joshi et al. 2018).

An alternative mechanism how protein aggregation might be linked to tRNA modification defects causing ribosomal pausing lies with disturbance of co-translational protein folding (Nedialkova and Leidel 2015). Support for this assumption stems from the observation of similarities in protein aggregate induction in a $\mathrm{mcm}^{5} \mathrm{~s}^{2} \mathrm{U}$-deficient yeast strain and a mutant lacking the ribosome-associated chaperones Ssb1/2, which are important for co-translational protein folding (Nedialkova and Leidel 2015). Thus, multiple mechanisms might link tRNA modification defects to the production of faulty proteins, which may be relevant for the common observation of impaired protein homeostasis in different tRNA modification mutants (Klassen et al. 2016; Nedialkova and Leidel 2015; Pollo-Oliveira et al. 2020; Thiaville et al. 2016; Xu et al. 2019).

\section{Starvation responses of tRNA modification mutants}

Interestingly, different tRNA modification defects also evoke major transcriptome changes and part of these are reminiscent of the transcriptomic response to nutrient depletion. Several yeast tRNA modification mutants including those lacking $\mathrm{mcm}^{5} \mathrm{~s}^{2} \mathrm{U}$ and $\mathrm{ct}^{6} \mathrm{~A}$ induce GCN4-dependent amino acid biosynthesis genes despite the presence of amino acids in the medium (Daugeron et al. 2011; Zinshteyn and Gilbert 
2013). In absence of either $\mathrm{mcm}^{5} \mathrm{~s}^{2} \mathrm{U}$ or $\mathrm{ct}^{6} \mathrm{~A}, G C N 4$ induction occurred independent of the Gcn2 kinase which is activated upon binding of uncharged tRNA (Daugeron et al. 2011; Zinshteyn and Gilbert 2013). The GCN2-independent GCN4 induction in these mutants suggested a non-canonical mechanism is involved in expression of general amino acid control (GAAC) genes in different tRNA modification mutants (Daugeron et al. 2011; Zinshteyn and Gilbert 2013). The recent characterization of transcriptomic changes after combined loss of $\mathrm{mcm}^{5} \mathrm{~s}^{2} \mathrm{U}$ and either $\mathrm{ct}^{6} \mathrm{~A}$ or $\Psi_{38 / 39}$ revealed additional facets of a common starvation in response to loss of different tRNA modifications (Bruch et al. 2020).

In these strains, when grown to early exponential phase, premature transcriptional activation of genes occurred that are normally expressed only upon entry into stationary phase or nutrient depletion. This includes a loss of glucose repression and induction of nitrogen catabolite-repressed (NCR) genes in addition to the activation of different amino acid biosynthesis genes (Bruch et al. 2020). Also, autophagy (another cellular starvation response) was induced as judged from studying loss of Atg13 phosphorylation and degradation of a GFP-Atg8 fusion protein. Since NCR and autophagy are controlled by the TORC 1 complex in budding yeast, these cellular responses to combined tRNA modification defects might be caused by loss or suppression of TORC1 activity (Bruch et al. 2020). Additional evidence for a role of Elp3-dependent tRNA modification in reciprocal regulation of TORC 1 and TORC 2 activities was obtained in a recent fission yeast study (Candiracci et al. 2019). In budding yeast, TORC1 activity also appears to be influenced by the level of uncharged tRNAs (Kamada 2017). These results suggest that the TOR complex, which represents a master regulator of growth and metabolism (Loewith and Hall 2011) might monitor the modification and charging status of tRNA. Loss of $\mathrm{mcm}^{5} \mathrm{U}$ or $\mathrm{s}^{2} \mathrm{U}$ modifications not only influences nutrient sensitive gene expression signatures, but also results in robust changes in cellular metabolism, and some of these are again reminiscent of cellular responses to nutrient starvation (Gupta et al. 2019; Karlsborn et al. 2016). Thus, apart from tRNA aminoacylation, multiple lines of evidence support an emerging role for tRNA anticodon loop modifications in the cellular signaling of nutrient availability.

\section{Potential mediators of nutrient signaling defects in tRNA modification mutants}

Several tRNA modification defects in yeast are known to trigger GCN4 expression in the absence of amino acid starvation. This includes not only the $\mathrm{mcm}^{5} \mathrm{~s}^{2} \mathrm{U}$ and $\mathrm{ct}^{6} \mathrm{~A}$ defective mutants described above, but was also observed in degl, pus7, rit1, trml, trm 7, $\bmod 5$ and tyw 3 mutants lacking various other tRNA modifications (Chou et al. 2017;
Han et al. 2018). While such amino acid starvation response appeared to be independent of the Gcn 2 kinase responding to uncharged tRNA in $\mathrm{mcm}^{5} \mathrm{~s}^{2} \mathrm{U}$ and $\mathrm{ct}^{6} \mathrm{~A}$ defective strains, it was shown to be Gcn2 dependent in trm 7 mutants (Daugeron et al. 2011; Han et al. 2018; Zinshteyn and Gilbert 2013). In these mutants, which lack $2^{\prime}-O$-methylation of $\mathrm{C} 32$ and G34 in tRNA ${ }^{\text {Phe }}$, reduced charging of the hypomodified tRNA was observed (Han et al. 2018). Hence, the GAAC starvation response in tRNA modification mutants can be triggered in some cases by effects on the tRNA aminoacylation efficiency.

In $\mathrm{s}^{2} \mathrm{U}$-deficient strains, robust metabolic changes involve increased storage carbohydrate synthesis, which normally occurs after glucose depletion (Gupta et al. 2019). Interestingly, these effects were linked to a disturbance of phosphate homeostasis. Increased trehalose synthesis likely occurs to counteract reduced intracellular phosphate levels since trehalose generation from trehalose phosphate can replenish intracellular phosphate levels. The phosphate shortage in $\mathrm{s}^{2} \mathrm{U}$-deficient mutants is thought to be triggered by transcriptional and translational downregulation of $\mathrm{PHO}$ genes involved in phosphate uptake (Gupta et al. 2019). A similar mechanism might be involved in starvation like responses in other tRNA modification mutants, including those required for formation of $\mathrm{mcm}^{5} \mathrm{U}$ and $\mathrm{ct}^{6} \mathrm{~A}$, since transcriptional downregulation of $\mathrm{PHO}$ genes was observed (Chou et al. 2017). In the $s^{2} U$-deficient strain, however, no robust transcriptional starvation response was triggered (Gupta et al. 2019), which is in contrast to the changes seen in combined mutants. Since the combined mutants exhibit growth defects exceeding those of the $\mathrm{s}^{2} \mathrm{U}$-deficient strain, more robust changes might occur also at the metabolic level (Bruch et al. 2020; Klassen et al. 2016). It remains unknown, however, how exactly the transcriptional response is mediated.

Intriguingly, when studying the transcriptional induction of nutrient responsive genes in combined tRNA modification mutants, their expression was dampened upon overexpression of the very same tRNAs that conferred a suppression of growth defects (Bruch et al. 2020; Klassen et al. 2016). As outlined above, the overexpressed tRNA presumably directly counteracts the inefficiency in decoding. At the same time, the propensity to accumulate protein aggregates (see above) is significantly lowered by the tRNA overexpression constructs. Hence, protein aggregates are linked to the decoding defect and are potentially involved in the observed gene expression changes (Fig. 1). Further support for this hypothesis was obtained from studying a mutant (zuol) accumulating protein aggregates independent of a tRNA modification defect (Bruch et al. 2020). In zuol mutants, the ribosome-associated chaperone system is severely compromised, leading to accumulation of protein aggregates (Bruch et al. 2020). At the same time, marker genes that are subject to glucose repression or NCR become transcriptionally 
induced despite the presence of glucose and ammonia in the medium. Thus, protein aggregates might be mechanistically involved in mediating transcriptional changes in response to combined loss of tRNA modifications. Possibly, the proteasome-mediated turnover of normally short-lived transcription factors is altered upon cellular accumulation of protein aggregates, ultimately leading to the observed changes in gene expression signatures. Further work will be required to test this hypothesis and other potentially involved mechanisms.

Acknowledgements We gratefully acknowledge support from the Deutsche Forschungsgemeinschaft (DFG) to R.S. (SCHA750/15-2) and their Priority Program SPP1784 Chemical Biology of Native Nucleic Acid Modifications to R.S. (SCHA750/20-2) and R.K. (KL2937/1-2).

Funding This work was supported by Deutsche Forschungsgemeinschaft (DFG) Grants SCHA750/15-2, SCHA750/20-2 to R.S. and KL2937/1-2 to R.K. Open Access funding provided by Projekt DEAL.

\section{Compliance with ethical standards}

Conflict of interest Not applicable.

Open Access This article is licensed under a Creative Commons Attribution 4.0 International License, which permits use, sharing, adaptation, distribution and reproduction in any medium or format, as long as you give appropriate credit to the original author(s) and the source, provide a link to the Creative Commons licence, and indicate if changes were made. The images or other third party material in this article are included in the article's Creative Commons licence, unless indicated otherwise in a credit line to the material. If material is not included in the article's Creative Commons licence and your intended use is not permitted by statutory regulation or exceeds the permitted use, you will need to obtain permission directly from the copyright holder. To view a copy of this licence, visit http://creativecommons.org/licenses/by/4.0/.

\section{References}

Agris PF (2008) Bringing order to translation: the contributions of transfer RNA anticodon-domain modifications. EMBO Rep 9:629-635. https://doi.org/10.1038/embor.2008.104

Björk GR, Huang B, Persson OP, Byström AS (2007) A conserved modified wobble nucleoside (mcm5s2U) in lysyl-tRNA is required for viability in yeast. RNA 13:1245-1255. https://doi.org/10.1261/ rna.558707

Bruch A, Laguna T, Butter F, Schaffrath R, Klassen R (2020) Misactivation of multiple starvation responses in yeast by loss of tRNA modifications. Nucleic Acids Res. https://doi.org/10.1093/nar/ gkaa455

Candiracci J, Migeot V, Chionh Y-H, Bauer F, Brochier T, Russell B, Shiozaki K, Dedon P, Hermand D (2019) Reciprocal regulation of TORC signaling and tRNA modifications by Elongator enforces nutrient-dependent cell fate. Sci Adv. https://doi.org/10.1126/ sciadv.aav0184

Chou H-J, Donnard E, Gustafsson HT, Garber M, Rando OJ (2017) Transcriptome-wide analysis of roles for tRNA modifications in translational regulation. Mol Cell 68:978-992.e4. https://doi. org/10.1016/j.molcel.2017.11.002
Daugeron M-C, Lenstra TL, Frizzarin M, El Yacoubi B, Liu X, BaudinBaillieu A, Lijnzaad P, Decourty L, Saveanu C, Jacquier A, Holstege FCP, de Crécy-Lagard V, van Tilbeurgh H, Libri D (2011) Gcn 4 misregulation reveals a direct role for the evolutionary conserved EKC/KEOPS in the t6A modification of tRNAs. Nucleic Acids Res 39:6148-6160. https://doi.org/10.1093/nar/gkr178

Gupta R, Walvekar AS, Liang S, Rashida Z, Shah P, Laxman S (2019) A tRNA modification balances carbon and nitrogen metabolism by regulating phosphate homeostasis. Elife. https://doi.org/10.7554/ eLife.44795

Han L, Kon Y, Phizicky EM (2015) Functional importance of $\Psi 38$ and I39 in distinct tRNAs, amplified for tRNAGln(UUG) by unexpected temperature sensitivity of the s2U modification in yeast. RNA 21:188-201. https://doi.org/10.1261/rna.048173.114

Han L, Guy MP, Kon Y, Phizicky EM (2018) Lack of 2'-O-methylation in the tRNA anticodon loop of two phylogenetically distant yeast species activates the general amino acid control pathway. PLoS Genet 14:e1007288. https://doi.org/10.1371/journal.pgen.10072 88

Huang B, Johansson MJO, Byström AS (2005) An early step in wobble uridine tRNA modification requires the Elongator complex. RNA 11:424-436. https://doi.org/10.1261/rna.7247705

Johansson MJO, Xu F, Byström AS (2018) Elongator-a tRNA modifying complex that promotes efficient translational decoding. Biochim Biophys Acta Gene Regul Mech 1861:401-408. https://doi. org/10.1016/j.bbagrm.2017.11.006

Joshi K, Bhatt MJ, Farabaugh PJ (2018) Codon-specific effects of tRNA anticodon loop modifications on translational misreading errors in the yeast Saccharomyces cerevisiae. Nucleic Acids Res. https://doi.org/10.1093/nar/gky664

Kamada Y (2017) Novel tRNA function in amino acid sensing of yeast Tor complex1. Genes Cells 22:135-147. https://doi.org/10.1111/ gtc. 12462

Karlsborn T, Mahmud AKMF, Tükenmez H, Byström AS (2016) Loss of ncm5 and mcm5 wobble uridine side chains results in an altered metabolic profile. Metabolomics 12:177. https://doi.org/10.1007/ s11306-016-1120-8

Khonsari B, Klassen R (2020) Impact of Pus1 pseudouridine synthase on specific decoding events in Saccharomyces cerevisiae. Biomolecules. https://doi.org/10.3390/biom10050729

Klassen R, Grunewald P, Thüring KL, Eichler C, Helm M, Schaffrath $\mathrm{R}$ (2015) Loss of anticodon wobble uridine modifications affects tRNA(Lys) function and protein levels in Saccharomyces cerevisiae. PLoS ONE 10:e0119261. https://doi.org/10.1371/journ al.pone. 0119261

Klassen R, Ciftci A, Funk J, Bruch A, Butter F, Schaffrath R (2016) tRNA anticodon loop modifications ensure protein homeostasis and cell morphogenesis in yeast. Nucleic Acids Res 44:1094610959. https://doi.org/10.1093/nar/gkw705

Klassen R, Bruch A, Schaffrath R (2017) Independent suppression of ribosomal +1 frameshifts by different tRNA anticodon loop modifications. RNA Biol 14:1252-1259. https://doi.org/10.1080/15476 286.2016.1267098

Leidel S, Pedrioli PGA, Bucher T, Brost R, Costanzo M, Schmidt A, Aebersold R, Boone C, Hofmann K, Peter M (2009) Ubiquitinrelated modifier Urm1 acts as a sulphur carrier in thiolation of eukaryotic transfer RNA. Nature 458:228-232. https://doi. org/10.1038/nature07643

Loewith R, Hall MN (2011) Target of rapamycin (TOR) in nutrient signaling and growth control. Genetics 189:1177-1201. https:// doi.org/10.1534/genetics.111.133363

Miyauchi K, Kimura S, Suzuki T (2013) A cyclic form of N6-threonylcarbamoyladenosine as a widely distributed tRNA hypermodification. Nat Chem Biol 9:105-111. https://doi.org/10.1038/nchem bio. 1137 
Nedialkova DD, Leidel SA (2015) Optimization of codon translation rates via tRNA modifications maintains proteome integrity. Cell 161:1606-1618. https://doi.org/10.1016/j.cell.2015.05.022

Pollo-Oliveira L, Klassen R, Davis N, Ciftci A, Bacusmo JM, Martinelli M, DeMott MS, Begley TJ, Dedon PC, Schaffrath R, de Crécy-Lagard V (2020) Loss of elongator- and KEOPS-dependent tRNA modifications leads to severe growth phenotypes and protein aggregation in yeast. Biomolecules. https://doi.org/10.3390/ biom 10020322

Ranjan N, Rodnina MV (2017) Thio-Modification of tRNA at the wobble position as regulator of the kinetics of decoding and translocation on the ribosome. J Am Chem Soc 139:5857-5864. https://doi. org/10.1021/jacs.7b00727

Rezgui VAN, Tyagi K, Ranjan N, Konevega AL, Mittelstaet J, Rodnina MV, Peter M, Pedrioli PGA (2013) tRNA tKUUU, tQUUG, and tEUUC wobble position modifications fine-tune protein translation by promoting ribosome A-site binding. Proc Natl Acad Sci U S A 110:12289-12294. https://doi.org/10.1073/pnas.1300781110

Schaffrath R, Leidel SA (2017) Wobble uridine modifications-a reason to live, a reason to die?! RNA Biol 14:1209-1222. https://doi. org/10.1080/15476286.2017.1295204

Sokołowski M, Klassen R, Bruch A, Schaffrath R, Glatt S (2017) Cooperativity between different tRNA modifications and their modification pathways. Biochim Biophys Acta. https://doi.org/10.1016/j. bbagrm.2017.12.003

Thiaville PC, Iwata-Reuyl D, de Crécy-Lagard V (2014) Diversity of the biosynthesis pathway for threonylcarbamoyladenosine $(\mathrm{t}(6)$ A), a universal modification of tRNA. RNA Biol 11:1529-1539. https://doi.org/10.4161/15476286.2014.992277

Thiaville PC, Legendre R, Rojas-Benítez D, Baudin-Baillieu A, Hatin I, Chalancon G, Glavic A, Namy O, de Crécy-Lagard V (2016)
Global translational impacts of the loss of the tRNA modification t6A in yeast. Microb Cell 3:29-45. https://doi.org/10.15698 /mic2016.01.473

Tükenmez H, Xu H, Esberg A, Byström AS (2015) The role of wobble uridine modifications in +1 translational frameshifting in eukaryotes. Nucleic Acids Res 43:9489-9499. https://doi.org/10.1093/ nar/gkv832

Väre VYP, Eruysal ER, Narendran A, Sarachan KL, Agris PF (2017) Chemical and conformational diversity of modified nucleosides affects tRNA structure and function. Biomolecules. https://doi. org/10.3390/biom7010029

Vendeix FAP, Murphy FV, Cantara WA, Leszczyńska G, Gustilo EM, Sproat B, Malkiewicz A, Agris PF (2012) Human tRNA(Lys3) (UUU) is pre-structured by natural modifications for cognate and wobble codon binding through keto-enol tautomerism. J Mol Biol 416:467-485. https://doi.org/10.1016/j.jmb.2011.12.048

Xu F, Byström AS, Johansson MJO (2019) SSD1 suppresses phenotypes induced by the lack of Elongator-dependent tRNA modifications. PLoS Genet 15:e1008117. https://doi.org/10.1371/journ al.pgen. 1008117

Zinshteyn B, Gilbert WV (2013) Loss of a conserved tRNA anticodon modification perturbs cellular signaling. PLoS Genet 9:e1003675. https://doi.org/10.1371/journal.pgen.1003675

Publisher's Note Springer Nature remains neutral with regard to jurisdictional claims in published maps and institutional affiliations. 